\title{
EDITORIAL MESSAGE
}

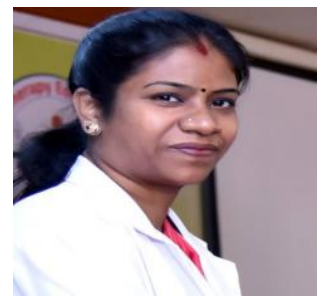

World AIDS Day, designated on 1 December every year since 1988, is dedicated to raising awareness of the AIDS pandemic caused by the spread of VIV infection, and mourning those who have died of the disease. Government and health officials, nongovernmental organization and individuals around the world observe the day, often with education on AIDS prevention and control. UNAIDS created the World AIDS campaign in 1997 to focus on year-round communications, prevention and education. In 2004, the World AIDS Campaign became an independent organization. In 2015 about 36.7 million people were living with HIV and its resulted in 1.1 million deaths. Most of those infected live in sub-saharan Africa. Between its discovery and 2014 AIDS has caused an estimated 39 million deaths worldwide. HIV/AIDS is considered a pandemic-a disease outbreak which is present over a large area and is actively spreading. HIV is believed to have originated in west-central Africa during the late $19^{\text {th }}$ or early $20^{\text {th }}$ century. AIDS was first recognized by the United States Centers for Disease Control and Prevention (CDC) in 1981 and its cause - HIV infection-was identified in the early part of the decade. HIV is transmitted by three main routes: sexual contact, significant exposure to infected body fluids or tissues and from mother to child during pregnancy, delivery, or breastfeeding (known as vertical transmission). There is no of acquiring HIV if exposed to feces, nasal secretions, saliva, sputum, sweat, tears, urine, or vomit unless these are contaminated with blood. It is possible to be co-infected by more than one strain of HIV-a condition known as HIV super infection. 
Human immunodeficiency virus and acquired immune deficiency syndrome (HIV/AIDS) is a spectrum of conditions caused by induction with the human immunodeficiency virus (HIV). Following initial infection, a person may not notice any symptoms or may experience a brief period of influenza-like illness. Typically, this is followed by prolonged period with no symptoms. As the infection progresses, it interferes more with the immune system, increasing the risk of common infections like tuberculosis, as well as other opportunistic infections, and tumors that rarely affect people who have working immune systems. These late symptoms of infection are referred to as AIDS. This stage is often also associated with weight loss.

As of 2013, AIDS has killed more than 36 million people worldwide (1981-2012), and an estimated 35.3 million people are living with HIV, making it one of the most importuned global public health issues in recorded history. Despite recent improved access to antiretroviral treatment in many regions of the world, the AIDS epidemic claims an estimated 2 million lives each year, of which about 2,70,000 are children.

There is currently no cure or effective HIV vaccine. Treatment consists of highly active antiretroviral therapy (HAART) which slows progression of the disease. As of 2010 more than 6.6 million people were taking them in low and middle income countries. Treatment also includes preventive and active treatment of opportunistic infections.

\section{Dr.Renuka.K}

Editor In Chief 